\title{
MODELING THE CONFORMALITY OF ATOMIC LAYER DEPOSITION: THE EFFECT OF STICKING PROBABILITY
}

\author{
Jolien Dendooven, Jan Musschoot, Davy Deduytsche, Roland Vanmeirhaeghe and \\ Christophe Detavernier \\ Department of Solid State Sciences, Ghent University, Krijgslaan 281 (S1), B-9000 Gent, \\ Belgium \\ email: Jolien.Dendooven@UGent.be
}

Atomic layer deposition (ALD) is known to be an excellent technique for conformal coating. In this work, two models, a kinetic and a Monte Carlo model, are developed to predict the deposited film thickness as a function of depth inside a hole. Earlier work by Gordon et al. assumed a sticking probability of $0 / 100 \%$ for molecules hitting a covered/uncovered section of the wall of the hole, thus resulting in a stepwise coverage profile after a single ALD cycle [1]. However, experimental studies indicate a gradual decrease of film thickness instead of a stepwise drop (figure) [2, and references therein]. It has been argued that the gradual slope may be related to (i) the increasing aspect ratio (AR) during deposition and (ii) the sticking probability, which is less than $100 \%$. The first explanation seems reasonable for the case of microscopic trenches, because during each cycle the deposition of new material results in a decrease of the diameter of the hole, and consequently the effective aspect ratio increases during deposition. However, our experiments using macroscopic structures $(\sim 0.1 \times 5 \times 20 \mathrm{~mm})$ also show a gradual decrease (figure), suggesting that the sticking probability is an important parameter for predicting the conformality. Therefore, in both models, we related the sticking probability $s$ to the surface coverage $\theta$ by Langmuir's equation $s(\theta)=s_{0}(1-\theta)$, whereby the initial sticking probability $s_{0}$ is now an adjustable model parameter. For $s_{0} \approx 100 \%$, the models predict a steplike profile, in agreement with Gordon et al., while for smaller values of $\mathrm{s}_{0}$, a gradual decreasing coverage profile is predicted. The two models show a good correspondence and follow the same trends as the experimental data (figure).
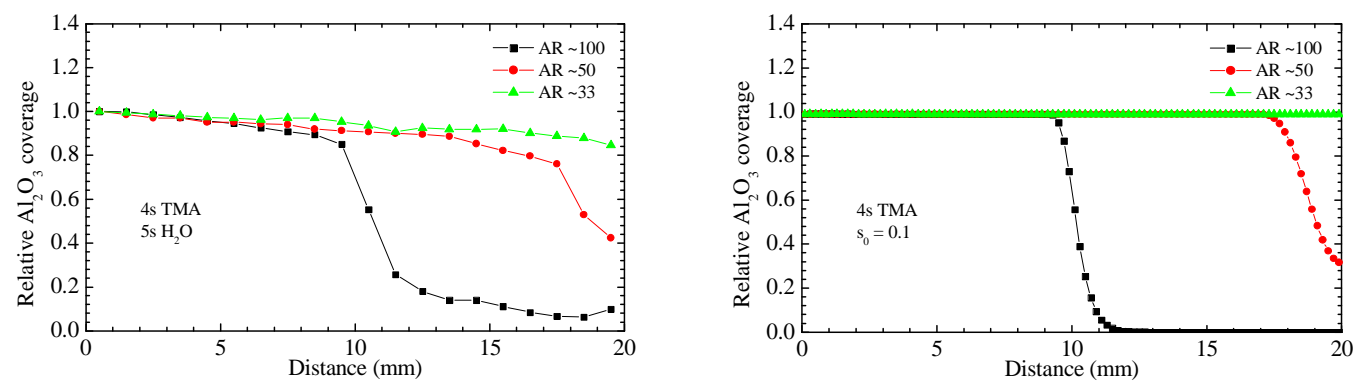

Figure: $\mathrm{Al}_{2} \mathrm{O}_{3}$ coverage profiles in macroscopic holes using $\mathrm{SiO}_{2}$ substrates (left), and simulation results for $s_{0}=0.1$ using the proposed kinetic model (right) [2].

[1] R. G. Gordon, D. Hausmann, E. Kim, J. Shepard, Chem. Vap. Deposition 9, 73(2003) [2] J. Dendooven, D. Deduytsche, J. Musschoot, R. L. Vanmeirhaeghe, C. Detavernier, J. Electrochem. Soc. 156, P63(2009) 